\title{
Neurologists' diagnostic accuracy of depression and cognitive problems in patients with parkinsonism
}

\author{
Angela EP Bouwmans ${ }^{1 *}$ and Wim EJ Weber ${ }^{1,2}$
}

\begin{abstract}
Background: Depression and cognitive impairment $(\mathrm{Cl})$ are important non-motor symptoms in Parkinson's Disease (PD) and related syndromes, but it is not clear how well they are recognised in daily practice. We have studied the diagnostic performance of experienced neurologists on the topics depression and cognitive impairment during a routine encounter with a patient with recent-onset parkinsonian symptoms.

Methods: Two experienced neurologists took the history and examined 104 patients with a recent-onset parkinsonian disorder, and assessed the presence of depression and cognitive impairment. On the same day, all patients underwent a Hamilton Depression Rating Scale test, and a Scales for Outcomes in Parkinson's DiseaseCognition-test (SCOPA-COG).
\end{abstract}

Results: The sensitivity of the neurologists for the topic depression was poor: $33.3 \%$. However, the specificity varied from 90.8 to $94.7 \%$. The patients' sensitivity was higher, although the specificity was lower. On the topic $\mathrm{Cl}$, the sensitivity of the neurologists was again low, in a range from 30.4 up to 34.8\%: however the specificity was high, with $92.9 \%$. The patients' sensitivity and specificity were both lower, compared to the number of the neurologists.

Conclusions: Neurologists' intuition and clinical judgment alone are not accurate for detection of depression or cognitive impairment in patients with recent-onset parkinsonian symptoms because of low sensitivity despite of high specificity.

Trial registration: (ITRSCC)NCT0036819.

Keywords: Mood disorder, Dementia, Non-motor, Parkinson's disease

\section{Background}

Depression and cognitive impairment $(\mathrm{CI})$ are increasingly appreciated as important non-motor symptoms in patients with Parkinson's disease (PD) and related syndromes [1-11]. Early recognition and diagnosis of both is important as treatment may increase quality of life of patients [12-22], but this is reported to be hampered at different levels. Many depressed patients are not aware of their problems [23] and, although caregivers sometimes have better judgment [24], they can also be misled by personality issues [25]. Non-psychiatrist physicians

\footnotetext{
*Correspondence: A.Bouwmans@mumc.nl

'Department of Neurology, Maastricht University Medical Centre, PO Box 5800 Maastricht, AZ 6202, Netherlands

Full list of author information is available at the end of the article
}

appear not to do much better, as numerous studies have shown that they perform significantly worse than validated questionnaires [26-39]. The presence of CI also leads physicians to overestimate the presence of depression [40].

Non-motor symptoms in PD tend to be underdiagnosed compared to motor problems, which seems logical as the latter are the primary expertise of neurologists. Some studies have compared different instruments to diagnose CI and depression in PD [41-43], but few have studied how accurate the diagnostic process is in normal daily practice $[44,45]$. Most often, neurologists focus on motor symptoms and evaluate possible $\mathrm{CI}$ and depression implicitly during their consultation [46]. These consultations are often too short to conduct a formal validated test for depression and/or CI. We studied

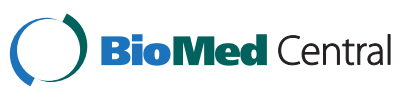


this implicit diagnostic process to assess its accuracy in a consecutive series of patients referred for analysis of a parkinsonian disorder of very recent onset. We focused on this patient group as research on this question has hitherto been done only in patients with a wellestablished diagnosis of PD.

\section{Methods}

The present study was nested in a larger, prospective study testing the diagnostic accuracy of transcranial duplex scanning (TCD) of the substantia nigra $(\mathrm{SN})$ in the brainstem as an instrument to diagnose $\mathrm{PD}$ in patients with a parkinsonism of unclear origin [47].

We invited 283 consecutive patients, who were referred to two neurology outpatient clinics, for analysis of clinically unclear parkinsonian disorder (Neurology Outpatient Clinics of the Maastricht University Medical Centre in Maastricht and of the Maasland Hospital in Sittard, both in The Netherlands). Patients, whose parkinsonism was clearly diagnosable at the first visit, were excluded from the study. For further details see our protocol described elsewhere [47]. Finally, we enrolled 242 patients in our study (see Figure 1) after written informed consent for participation by each patient.

After two years, all patients were re-examined by a pair of neurologists for a final clinical diagnosis. The neurologists were specialists in movement disorders, with more than ten years' experience in this field. These investigators were blinded for all prior test results from these patients. In planning these visits, we made sure that neither of the two neurologists had ever seen the patient. They were asked to interview and examine the patient, guided by a standard form (see Additional file 1 ). Family or spouse were allowed to be present at this consultation, but were asked to refrain from answering any questions. Neurologists were not given any objective scales for depression or cognitive impairment. After this consultation, the patient left the room.

The specialists were then asked to indicate on a form if they thought the patient was depressed and if CI was present. There was no communication between the raters on this decision. They were also asked to reach an independent clinical diagnosis, and record it. After this, they were asked to discuss the patient, and reach a final, consensus diagnosis, which served as the "gold standard" in the TCD study [47]. On the day of this evaluation, all patients were asked to complete the Hamilton Depressing Rating Scale (in the text further shortened to 'Hamilton') and the Scales for Outcomes in Parkinson's Disease-Cognition (SCOPA-COG). Both tests have shown their reliability and validity as instruments to assess either depression or $\mathrm{CI}$, both with and without PD [48-53]. The maximum possible score on the SCOPACOG is 43. A score of 20 or lower is defined as CI

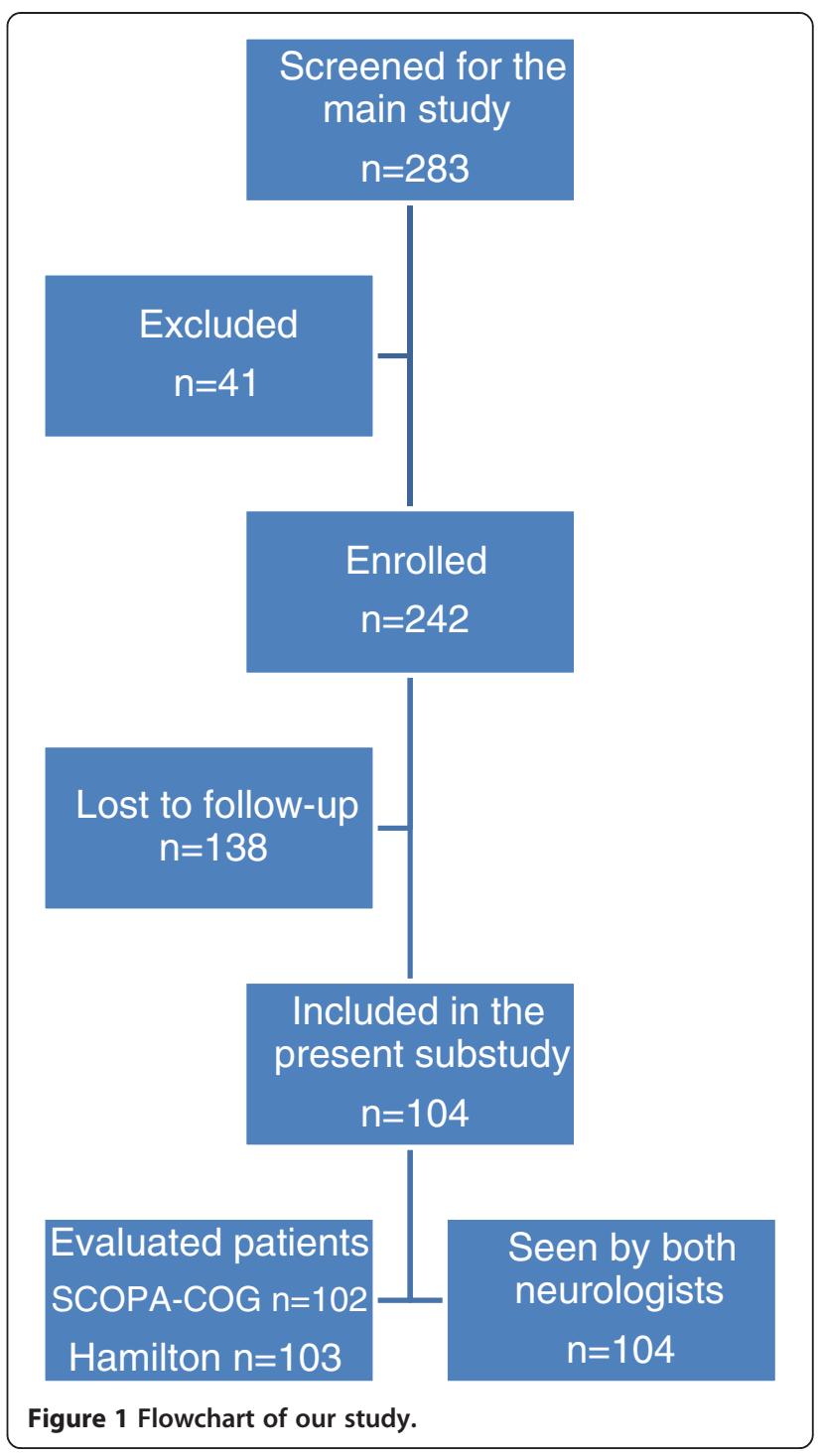

[52,53]. The maximum possible score on the 17 item Hamilton is 52. A score 0 to 7 on the Hamilton implies normal/-borderline mood, score between 8 and 15 indicates a mild depression; a score in the range of 16 to 26 indicates a moderate depression; and a score of 27 or higher implies severe depression [50,51]. The results of these tests were not provided to the patients. We also asked the patients the following questions: 'Do you think you have more difficulties with your memory than people of the same age as you?' and 'Did you experience feelings of depression, most of the time during the last month?' to evaluate their insight about the presence of $\mathrm{CI}$ and depression.

The primary objective of this study was to define the accuracy, namely, the sensitivity and specificity of the neurologists' clinical judgement regarding the presence of $\mathrm{CI}$ and depression. The secondary objective was to evaluate the degree of agreement on the diagnosis of CI 
and depression between the patient himself and the two neurologists. SPSS 16.0 for windows (SPSS, Chicago, IL, USA) was used for statistical analysis. The accuracy of the clinical judgement of the neurologists was evaluated by the means of specificity and sensitivity. The inter-rater agreement was evaluated by the Kappa statistics.

\section{Results}

We had originally enrolled 242 patients into the TCD study (see Figure 1). Follow-up after two years took place between September 2008 and September 2010. Thirty patients (12,4\%) had died and 108 patients $(44,2 \%)$ were unable or unwilling to undergo a second evaluation. The group lost to follow up were significantly older $(p=0,034)$ and had a higher mean Unified Parkinson's Disease Rating Scale (UPDRS) at enrollment $(p=0,031)$. However there were no significant differences in the distribution of the final diagnoses compared to the patients included in the present study. No correlation was found between either the UPDRS scores or results on the Hamilton and SCOPA-COG and the final diagnoses after two years follow-up.

For the present study, 104 patients $(65$ male, 39 female) were evaluated. The mean age was 70,3 (range 44-90) years. After two years follow-up, $62,5 \%$ of the patients used antiparkinson medication, $13,5 \%$ antidepressants, and 3,8\% neuroleptics. No one used cognitive enhancers. The final clinical diagnosis was PD in 53 (51\%) patients. For further patient characteristics, see Table 1. The diagnostic groups were demographically similar. The remaining 15 (14\%) patients without parkinsonism had alternative diagnoses, such as, isolated tremor, orthostatic tremor, tardive dyskinesia, multiinfarct dementia, Alzheimer disease, stroke, hypoxic encephalopathy, and psychogenic disorder.

\section{Cognitive impairment}

In total 102 patients were able to complete the SCOPACOG, two patients were too tired after the consultation with the neurologists, and therefore, were not able to complete this test. The mean SCOPA-COG score was 21,1 with a range of 4 to 36 . See Table 2 for the results on CI 'diagnoses' by the neurologist compared with the SCOPA-COG scores.

The sensitivity of neurologist 1 for 'diagnosing' CI was $34,8 \%$ and of neurologist $230,4 \%$ (see Table 3). Specificity of both neurologists was $92,9 \%$.

In answer to the question 'Do you think you have more difficulties with your memory than people of the same age as you?', 19 (18,6\%) said 'yes', 77 (75,5\%) said 'no', and $6(5,9 \%)$ 'I do not know'. See Table 2 for the results on this 'self-diagnosis' of the patients on CI combined with their SCOPA-COG scores. Because six patients could not answer the question with 'yes' or 'no', we excluded them from this analysis. Therefore the sensitivity of self-diagnosis of CI by the patient was $27,3 \%$. Its specificity was $86,5 \%$.

The agreement between neurologist 1 and neurologist 2 was good with a kappa value of 0,74 (95\% confidence interval 0,57-0,91) (see Table 3). However, the agreement between the neurologists and the patient was much lower with a kappa value varying between 0,34 (95\% confidence interval $0,12-0,56)$ and 0,44 (95\% confidence interval $0,23-0,66$ ).

\section{Depression}

In total, 103 patients were able to complete the Hamilton (one patient was too tired after the consultation and was not able to complete this test). The mean Hamilton score was 5,5 with a range of 0 to 26 . In this study, none of the patients had a score of 27 or higher. We defined depression at the total score of 8 or higher on the Hamilton.

Table 1 Patient characteristics (data as mean and SD) or count (\%)

\begin{tabular}{|c|c|c|c|c|c|c|c|}
\hline & $\begin{array}{l}\text { All patients } \\
(\mathrm{n}=104)\end{array}$ & $P D(n=53)$ & APS $(n=13)$ & VP $(n=8)$ & ET $(n=11)$ & DIP $(n=4)$ & $\begin{array}{l}\text { No parkinsonism } \\
(n=15)\end{array}$ \\
\hline Mean age in years (SD) & $70,3(9,46)$ & $69,81(9,74)$ & $70,29(7,42)$ & $73,71(1,60)$ & $67,91(10,38)$ & $67,50(12,61)$ & $72,44(11,06)$ \\
\hline Men & $64 \%$ & $63 \%$ & $75 \%$ & $56 \%$ & $91 \%$ & $50 \%$ & $47 \%$ \\
\hline Mean score UPDRS-III (SD) & $15,03(8,08)$ & $16,88(6,06)$ & $19,57(9,94)$ & $18,29(9,55)$ & $6,82(3,95)$ & $17,00(10,86)$ & $8,75(6,18)$ \\
\hline SCOPA-COG > 20 (no Cl) & $55 \%$ & $58 \%$ & $67 \%$ & $38 \%$ & $82 \%$ & $25 \%$ & $60 \%$ \\
\hline SCOPA-COG < =20 (yes Cl) & $45 \%$ & $42 \%$ & $33 \%$ & $62 \%$ & $18 \%$ & $75 \%$ & $40 \%$ \\
\hline Hamilton < 8 (no depression) & $74 \%$ & $81 \%$ & $54 \%$ & $71 \%$ & $73 \%$ & $100 \%$ & $60 \%$ \\
\hline Hamilton $>=8$ (yes depression) & $26 \%$ & $19 \%$ & $46 \%$ & $29 \%$ & $27 \%$ & $0 \%$ & $40 \%$ \\
\hline 'Self-diagnosed' presence of Cl & $38 \%$ & $34 \%$ & $69 \%$ & $25 \%$ & $18 \%$ & $50 \%$ & $47 \%$ \\
\hline 'Self-diagnosed' presence of depression & $18 \%$ & $13 \%$ & $23 \%$ & $13 \%$ & $36 \%$ & $25 \%$ & $20 \%$ \\
\hline
\end{tabular}

PD = Parkinson's disease, APS = atypical parkinsonian syndromes, VP= vascular parkinsonism, ET = essential tremor, DIP = drug induced parkinsonism, UPDRS-III = Unified Parkinson's Disease Rating Scale part III, SCOPA-COG = Scales for Outcomes in Parkinson's Disease-Cognition, Hamilton= Hamilton Depressing Rating Scale CI= cognitive impairment. 
Table 2 Results of neurologists'clinical assessment and patients' insight versus SCOPA-COG and Hamilton scores (data in numbers)

\begin{tabular}{|c|c|c|c|c|c|c|c|}
\hline & Rater 1 & Rater 1 & Rater 2 & Rater 2 & Patient & Patient & Patient \\
\hline & yes & no & yes & no & yes & no & do not know \\
\hline SCOPA-COG > 20 (no Cl) & 4 & 52 & 4 & 52 & 7 & 45 & 4 \\
\hline SCOPA-COG $<=20$ (yes Cl) & 16 & 30 & 14 & 32 & 12 & 32 & 2 \\
\hline Hamilton < 8 (no depression) & 7 & 69 & 4 & 72 & 21 & 49 & 6 \\
\hline Hamilton $>=8$ (yes depression) & 9 & 18 & 9 & 18 & 19 & 7 & 1 \\
\hline
\end{tabular}

SCOPA-COG = Scales for Outcomes in Parkinson's Disease-Cognition, Hamilton = Hamilton Depressing Rating Scale, $\mathrm{Cl}=$ cognitive impairment .

See Table 2 for the results on the depression diagnosed by the neurologists compared to the patients' Hamilton score. The sensitivity of depression diagnosis by both neurologists was $33,3 \%$, specificity of neurologist 1 was $90,8 \%$ and of neurologist 2 was $94,7 \%$ (see Table 3).

In answer to the question 'Did you experience feelings of depression, most of the time this last month?', $40(38,8 \%)$ said 'yes', $56(54,4 \%)$ said 'no', and 7 (6,8\%) 'I do not know'. See Table 2 for the results on 'selfdiagnosis' by the patients compared with their Hamilton score. Because 7 patients could not answer the question with 'yes' or 'no', we excluded them from this analysis. Therefore, the sensitivity of the patients' selfdiagnosis of depression was $73,1 \%$. Its specificity was $70,0 \%$.

The agreement between neurologist 1 and neurologist 2 was good with a kappa value of 0,62 (95\% confidence interval 0,39-0,85) (see Table 3). Agreement between neurologists and patients was lower, with a kappa value varying between 0,28 (95\% confidence interval $0,12-0,43)$ and $0,43(95 \%$ confidence interval $0,27-0,60)$.

Table 3 Summary of the neurologists' and patients' diagnostic performance

\begin{tabular}{lll}
\hline & Cl & Depression \\
\hline Rater 1 specificity & $92.9 \%$ & $90.8 \%$ \\
Rater 1 sensitivity & $34.8 \%$ & $33.3 \%$ \\
Rater 2 specificity & $92.9 \%$ & $94.7 \%$ \\
Rater 2 sensitivity & $30.4 \%$ & $33.3 \%$ \\
Agreement between & $0.74(0.57-0.91)$ & $0.62(0.39-0.85)$ \\
two neurologists kappa & & \\
$\begin{array}{l}\text { (95\% confidence interval) } \\
\text { Patient specificity }\end{array}$ & $86.5 \%$ & $70.0 \%$ \\
Patient sensitivity & $27.3 \%$ & $73.1 \%$ \\
$\begin{array}{l}\text { Neurologist 1-patient } \\
\text { agreement kappa }\end{array}$ & $0.34(0.12-0.56)$ & $0.43(0.27-0.60)$ \\
(95\% confidence interval) & & \\
$\begin{array}{l}\text { Neurologist 2-patient } \\
\text { agreement kappa }\end{array}$ & $0.44(0.23-0.66)$ & $0.28(0.12-0.43)$ \\
(95\% confidence interval) & & \\
\hline
\end{tabular}

\section{Discussion}

We studied the accuracy of neurologists' ability to diagnose depression and $\mathrm{CI}$ in patients with parkinsonian symptoms, in a way that most closely resembles normal daily clinical neurology practice, as a definite diagnosis of a parkinsonian syndrome is often not possible in the first few years. And, while the neurologists in our study were very experienced in PD and spent on average more time per patient than normal, we speculate that their results might even be somewhat inflated.

A limitation of the study is the use of psychometric scales as a proxy for the diagnoses of $\mathrm{CI}$ and depression. While these can not, of course, replace a complete diagnostic work-up by a specialised psychiatrist with a psychometric battery, we do feel that the results show that there is probably a considerable underestimation of the presence of these clinical problems also in patients with a very recent-onset of a parkinsonian disorder. Another limitation of our study is the large number of patients lost to follow-up. This could have biased our population towards one with less morbidity, thus increasing diagnostic difficulty. From the age and UPDRS-scores one can infer that PD patients with more severe disease and thus with possibly more severe depression and CI were underrepresented in this study.

We found that this implicit diagnostic process by neurologists is far less accurate than validated tests. The prevalences of both depression and CI found in our study, are representative for the general population of PD patients $[54,55]$.

We found that neurologists underestimated the number of patients with $\mathrm{CI}$, by up to $70 \%$. They did somewhat better than the patients themselves. Compared to other studies, our neurologists' diagnostic sensitivity for CI was lower than those of general practitioners (GP's), although both had a high specificity [34-40]. Our data on the recognition of $\mathrm{CI}$ are in line with two earlier studies on PD patients, which both found the majority of symptoms going unrecognised and untreated. However, one of those was a retrospective chart review and the other, prospective, study involved older, wellestablished PD patients. We did not confirm earlier 
research that the presence of $\mathrm{CI}$ leads to overestimating of depression by doctors [40].

In 'diagnosing' depression, our neurologists showed low sensitivity and high specificity. 'Self-diagnosis' of depression by the patient had a higher sensitivity compared to the neurologists, although the specificity was lower. The neurologists missed up to $67 \%$ of the patients with depression. The patients in our study overestimated the presence of depression. Presence of $\mathrm{CI}$ had no influence on the insight of the patients on depression. 'Diagnostic accuracy' of depression by our neurologists was comparable to GP's [26], but selfdiagnosis of depression in our patient population had a remarkably higher sensitivity than in an earlier study done by Watson [24]. However, that study only included patients with dementia, and this may explain this difference.

\section{Conclusions}

Intuition and clinical judgment are not enough for a neurologist to recognize depression and/or cognitive problems in patients with recent-onset parkinsonian syndromes, such as, PD and atypical parkinsonian syndromes (APS). It is important to realize this, considering the consequences of untreated depression and CI. Our neurologists had a high specificity diagnosing $\mathrm{CI}$ and depression, but at the same time missed more than half of the patients with these problems. Patients themselves are not better at selfdiagnosing these non-motor symptoms.

\section{Additional file}

Additional file 1: Standard clinical scorings form [2,33-38].

\section{Competing interest}

All authors declare that they have no competing interests.

\section{Authors' contribution}

Both authors have contributed to all of the following: 1. Research project: A. Conception, B. Organization, C. Execution; 2. Statistical Analysis: A. Design, B. Execution, C. Review and Critique; 3. Manuscript Preparation: A. Writing of the first draft, B. Review and Critique. Full financial disclosure for the previous 12 months: Bouwmans: Stichting Internationaal Parkinson Fonds. Weber: Stichting Internationaal Parkinson Fonds. Both authors read and approved the final manuscript.

\section{Acknowledgement}

This study was funded by the "Stichting Internationaal Parkinson Fonds". We thank consultant neurologists Bert Anten, Fred Vreeling, Annemarie Vlaar, and Ania Winogrodzka for their helpful cooperation.

\section{Author details}

'Department of Neurology, Maastricht University Medical Centre, PO Box 5800 Maastricht, AZ 6202, Netherlands. 'Division of Movement Disorders, Department of Neurology, Maastricht University Medical Centre, Maastricht, Netherlands.

Received: 17 November 2011 Accepted: 18 May 2012

Published: 15 June 2012

\section{References}

1. Reichmann H, Schneider C, Lohle M: Non-motor features of Parkinson's disease: depression and dementia. Parkinsonism Relat Disord 2009, 15 (Suppl 3):S87-S92.
2. Bassetti $\mathrm{CL}$ : Nonmotor Disturbances in Parkinson's Disease. Neurodegener Dis 2011, 8(3):95-108.

3. Chaudhuri KR, Odin P: The challenge of non-motor symptoms in Parkinson's disease. Prog Brain Res 2010, 184:325-341.

4. Chaudhuri KR, Naidu Y: Early Parkinson's disease and non-motor issues. J Neurol 2008, 255(Suppl 5):33-38.

5. Lohle M, Storch A, Reichmann H: Beyond tremor and rigidity: non-motor features of Parkinson's disease. J Neural Transm 2009, 116(11):1483-1492.

6. Park A, Stacy M: Non-motor symptoms in Parkinson's disease. J Neurol 2009, 256(Suppl 3):293-298.

7. Ziemssen T, Reichmann H: Non-motor dysfunction in Parkinson's disease. Parkinsonism Relat Disord 2007, 13(6):323-332.

8. Rodriguez-Violante $M$, et al: Prevalence of non-motor dysfunction among Parkinson's disease patients from a tertiary referral center in Mexico City. Clin Neurol Neurosurg 2010, 112(10):883-885.

9. Dickson DW, et al: Neuropathology of non-motor features of Parkinson disease. Parkinsonism Relat Disord 2009, 15(Suppl 3):S1-S5.

10. Colosimo $C$, et al: Non-motor symptoms in atypical and secondary parkinsonism: the PRIAMO study. J Neurol 2010, 257(1):5-14.

11. Kim YD, et al: Cognitive dysfunction in drug induced parkinsonism (DIP). Arch Gerontol Geriatr 2011, 53(2):e222-e226.

12. Gomez-Esteban JC, et al: Impact of psychiatric symptoms and sleep disorders on the quality of life of patients with Parkinson's diseas. J Neurol 2011, 258(3):494-499.

13. Weintraub D, et al: Effect of psychiatric and other nonmotor symptoms on disability in Parkinson's disease. J Am Geriatr Soc 2004, 52(5):784-788.

14. Carod-Artal FJ, et al: Anxiety and depression: main determinants of health-related quality of life in Brazilian patients with Parkinson's disease. Parkinsonism Relat Disord 2008, 14(2):102-108.

15. Carod-Artal FJ, Vargas AP, Martinez-Martin P: Determinants of quality of life in Brazilian patients with Parkinson's disease. Mov Disord 2007, 22(10):1408-1415.

16. Li H, et al: Nonmotor symptoms are independently associated with impaired health-related quality of life in Chinese patients with Parkinson's disease. Mov Disord 2010, 25(16):2740-2746.

17. Qin Z, et al: Health related quality of life in early Parkinson's disease: impact of motor and non-motor symptoms, results from Chinese levodopa exposed cohort. Parkinsonism Relat Disord 2009, 15(10):767-771.

18. Slawek J, Derejko M, Lass P: Factors affecting the quality of life of patients with idiopathic Parkinson's disease-a cross-sectional study in an outpatient clinic attendees. Parkinsonism Relat Disord 2005, 11(7):465-468

19. Martinez-Martin P, et al: The impact of non-motor symptoms on healthrelated quality of life of patients with Parkinson's disease. Mov Disord 2011, 26(3):399-406.

20. Schrag A, Jahanshahi M, Quinn N: What contributes to quality of life in patients with Parkinson's disease? J Neurol Neurosurg Psychiatry 2000, 69(3):308-312.

21. Klepac N, et al: Is quality of life in non-demented Parkinson's disease patients related to cognitive performance? A clinic-based cross-sectional study. Eur J Neurol 2008, 15(2):128-133.

22. Schrag $A$, et al: Health-related quality of life in multiple system atrophy. Mov Disord 2006, 21(6):809-815.

23. Arlt S, et al: The patient with dementia, the caregiver and the doctor: cognition, depression and quality of life from three perspectives. Int $J$ Geriatr Psychiatry 2008, 23(6):604-610.

24. Watson LC, et al: Perceptions of depression among dementia caregivers: findings from the CATIE-AD trial. Int J Geriatr Psychiatry 2011, 26(4):397-402.

25. Duberstein PR, et al: Detection of depression in older adults by family and friends: distinguishing mood disorder signals from the noise of personality and everyday life. Int Psychogeriatr 2011, 23(4):634-643.

26. Cepoiu M, et al: Recognition of depression by non-psychiatric physiciansa systematic literature review and meta-analysis. J Gen Intern Med 2008, 23(1):25-36.

27. Docherty JP: Barriers to the diagnosis of depression in primary care. J Clin Psychiatry 1997, 58(Suppl 1):5-10.

28. Wittchen HU, Pittrow D: Prevalence, recognition and management of depression in primary care in Germany: the Depression 2000 study. Hum Psychopharmacol 2002, 17(Suppl 1):S1-S11.

29. Parchman ML: Physicians' recognition of depression. Fam Pract Res J 1992, 12(4):431-438. 
30. Carney PA, et al: How physician communication influences recognition of depression in primary care. J Fam Pract 1999, 48(12):958-964.

31. Furedi J, et al: The role of symptoms in the recognition of mental health disorders in primary care. Psychosomatics 2003, 44(5):402-406.

32. Harman JS, et al: The effect of patient and visit characteristics on diagnosis of depression in primary care. J Fam Pract 2001, 50(12):1068

33. Luber MP, et al: Diagnosis, treatment, comorbidity, and resource utilization of depressed patients in a general medical practice. Int $J$ Psychiatry Med 2000, 30(1):1-13.

34. Pentzek $M$, et al: Apart from nihilism and stigma: what influences general practitioners' accuracy in identifying incident dementia? Am J Geriatr Psychiatry 2009, 17(11):965-975.

35. van Hout $\mathrm{H}$, et al: Are general practitioners able to accurately diagnose dementia and identify Alzheimer's disease? A comparison with an outpatient memory clinic. Br J Gen Pract 2000, 50(453):311-312.

36. Valcour VG, et al: The detection of dementia in the primary care setting. Arch Intern Med 2000, 160(19):2964-2968.

37. Chodosh J, et al: Physician recognition of cognitive impairment: evaluating the need for improvement. J Am Geriatr Soc 2004, 52(7):1051-1059.

38. O'Connor DW, et al: Do general practitioners miss dementia in elderly patients? BMJ 1988, 297(6656):1107-1110.

39. Lopponen $M$, et al: Diagnosing cognitive impairment and dementia in primary health care - a more active approach is needed. Age Ageing 2003, 32(6):606-612.

40. Crane MK, et al: Brief report: patient cognitive status and the identification and management of depression by primary care physicians. J Gen Intern Med 2006, 21(10):1042-1044.

41. Silberman $C D$, et al: Recognizing depression in patients with Parkinson's disease: accuracy and specificity of two depression rating scale. Ara Neuropsiquiatr 2006, 64(2B):407-411.

42. Tumas $V$, et al: The accuracy of diagnosis of major depression in patients with Parkinson's disease: a comparative study among the UPDRS, the geriatric depression scale and the Beck depression inventory. Arq Neuropsiquiatr 2008, 66(2A):6-152.

43. Leentjens AF, et al: The validity of the Hamilton and Montgomery-Asberg depression rating scales as screening and diagnostic tools for depression in Parkinson's disease. Int J Geriatr Psychiatry 2000, 15(7): 644-649.

44. Shulman LM, et al: Non-recognition of depression and other non-motor symptoms in Parkinson's disease. Parkinsonism Relat Disord 2002, 8(3): 193-197.

45. Burn DJ: Beyond the iron mask: towards better recognition and treatment of depression associated with Parkinson's disease. Mov Disord 2002, 17(3):445-454.

46. Gallagher DA, Lees AJ, Schrag A: What are the most important nonmotor symptoms in patients with Parkinson's disease and are we missing them? Mov Disord 2010, 25(15):2493-2500.

47. Vlaar AM, et al: Protocol of a prospective study on the diagnostic value of transcranial duplex scanning of the substantia nigra in patients with parkinsonian symptoms. BMC Neurol 2007, 7:28.

48. Reijnders JS, Lousberg R, Leentjens AF: Assessment of depression in Parkinson's disease: the contribution of somatic symptoms to the clinimetric performance of the Hamilton and Montgomery-Asberg rating scales. J Psychosom Res 2010, 68(6):561-565.

49. Schrag A, et al: Depression rating scales in Parkinson's disease: critique and recommendations. Mov Disord 2007, 22(8):1077-1092.

50. Furukawa TA: Assessment of mood: guides for clinicians. J Psychosom Res 2010, 68(6):581-589.

51. Furukawa TA, et al: Evidence-based guidelines for interpretation of the Hamilton Rating Scale for Depression. J Clin Psychopharmacol 2007, 27(5):531-534

52. Kulisevsky J, Pagonabarraga J: Cognitive impairment in Parkinson's disease: tools for diagnosis and assessment. Mov Disord 2009, 24(8): 1103-1110.

53. Carod-Artal FJ, et al: Psychometric attributes of the SCOPA-COG Brazilian version. Mov Disord 2008, 23(1):81-87.

54. Lemke MR: Depressive symptoms in Parkinson's disease. Eur J Neurol 2008, 15(Suppl 1):21-25.

55. Aarsland D, Kurz MW: The epidemiology of dementia associated with Parkinson's disease. Brain Pathol 2010, 20(3):633-639. doi:10.1186/1471-2377-12-37

Cite this article as: Bouwmans and Weber: Neurologists' diagnostic accuracy of depression and cognitive problems in patients with parkinsonism. BMC Neurology 2012 12:37.

\section{Submit your next manuscript to BioMed Central and take full advantage of:}

- Convenient online submission

- Thorough peer review

- No space constraints or color figure charges

- Immediate publication on acceptance

- Inclusion in PubMed, CAS, Scopus and Google Scholar

- Research which is freely available for redistribution 